\title{
IL-35, TNF- $\alpha$, BAFF, and VEGF serum levels in patients with different rheumatic diseases
}

\author{
Piotr Wojdasiewicz ${ }^{1}$, Anna Wajda², Ewa Haładyj ${ }^{3}$, Katarzyna Romanowska-Próchnicka ${ }^{3}$, \\ Anna Felis-Giemza ${ }^{3}$, Jolanta Nałęcz-Janik ${ }^{3}$, Marcela Walczyk ${ }^{3}$, Marzena Olesińska ${ }^{3}$, \\ Beata Tarnacka ${ }^{4}$, Agnieszka Paradowska-Gorycka ${ }^{2}$ \\ ${ }^{1}$ Department of Rehabilitation, National Institute of Geriatrics, Rheumatology and Rehabilitation, Warsaw, Poland \\ ${ }^{2}$ Department of Biochemistry and Molecular Biology, National Institute of Geriatrics, Rheumatology and Rehabilitation, \\ Warsaw, Poland \\ ${ }^{3}$ Department of Systemic Connective Tissue Diseases, National Institute of Geriatrics, Rheumatology and Rehabilitation, \\ Warsaw, Poland \\ ${ }^{4}$ Department of Rehabilitation, $1^{\text {st }}$ Faculty of Medicine, Medical University of Warsaw, Poland
}

\begin{abstract}
Objectives: Inflammatory processes in rheumatic diseases spread via various types of immune system cells and tissues with the aid of inflammatory cytokines and growth factors and the participation of vascular endothelium. Research is still conducted to determine the role of individual factors in the pathophysiology of rheumatic diseases. The task is complicated because the multiplane network of cytokines is characterized by complex correlations manifesting as positive and negative feedback, which impedes the definitive interpretation of the role of specific cytokines. Therefore, it seems justified to perform a comparative analysis of the expression of at least several molecules in one study, which may help reveal their role in the pathogenesis of rheumatic diseases and have prognostic value.

Material and methods: The aim of the study involves the assessment and comparative analysis of the concentrations of interleukin 35 (IL-35), tumour necrosis factor $\alpha$ (TNF- $\alpha$ ), B-cell-activating factor (BAFF), and vascular endothelial growth factor (VEGF) in peripheral blood serum in patients with rheumatoid arthritis (RA) ( $n=43)$, systemic lupus erythematosus (SLE) $(n=28)$, antiphospholipid syndrome (APS) $(n=24)$, and mixed connective tissue disease (MCTD) $(n=9)$. The main intention is to search for biomarkers for specific rheumatic diseases. Cytokine and growth factor levels were determined using specific ELISA kits.

Results: Statistically significant differences in VEGF and IL-35 concentrations occurred between patients with APS vs. RA and SLE vs. RA. There was a significant high positive correlation between the concentration of BAFF and TNF- $\alpha(r=0.77, p<0.0000)$ in patients with APS, as well as in patients with SLE $(r=0.55, p=0.00)$.

Conclusions: BAFF and TNF- $\alpha$ may be promising biomarkers in patients with APS and VEGF in patients with RA. Additionally, IL-35 may be a useful marker for the diagnosis of APS. Positive correlation of BAFF and TNF- $\alpha$ concentrations in APS and SLE potentially indicates much more similar etiopathogenesis of these diseases than it could be previously predicted.
\end{abstract}

Key words: cytokines, rheumatic diseases, BAFF, VEGF, biomarkers. 


\section{Introduction}

Rheumatic diseases constitute a large and diversified group of conditions with an underlying autoimmune cause. Their common feature is the imbalance in immune system homeostasis [1]. The hyperactivity of proinflammatory and catabolic processes may affect numerous internal organs and sites, with joints being the most commonly involved [2].

The pathophysiology of rheumatic diseases presents considerable similarities in many aspects, despite the heterogeneity of manifestations. Inflammatory processes spread via various types of immune system cells and tissues with the aid of inflammatory cytokines and growth factors and the participation of vascular endothelium (endothelium cells - ECs) [3].

Research is still conducted to determine the role of individual factors in the pathophysiology of rheumatic diseases. The task is complicated because the multiplane network of cytokines is characterized by complex correlations manifesting as positive and negative feedback, which impedes the definitive interpretation of the role of specific cytokines. Therefore, it seems justified to perform a comparative analysis of the expression of several molecules in one study, which may help reveal their role in the pathogenesis of rheumatic diseases and may have prognostic value.

The factors, whose influence on the development and course of rheumatic diseases is still undergoing research, include interleukin 35 (IL-35), tumour necrosis factor $\alpha$ (TNF- $\alpha$ ), B-cell-activating factor (BAFF), and vascular endothelial growth factor (VEGF). A similar function is performed by IL-35, which belongs to the interleukin 12 (IL-12) cytokine family. IL-35 is produced by CD4+ Foxp3+ regulatory T-cells (Tregs), B lymphocytes, and ECs [4]. The proinflammatory activity of IL-35 includes the stimulation of Treg proliferation and inhibition of the expression for anti-inflammatory interleukin 10 (IL-10) [4]. TNF- $\alpha$ is one of the best studied inflammatory cytokines, which stimulates numerous cell lines for immune response. Its role in the pathophysiology of autoimmune diseases is indisputable. Currently, modern biological treatment of the majority of rheumatic diseases is based on inhibiting the activity associated with the signaling pathway for TNF- $\alpha$ [5].

B-cell-activating factor is currently viewed as a very promising target in the treatment of rheumatic diseases. This cytokine is produced by macrophages, monocytes, ECs, or dendritic cells (DCs). It contributes to B lymphocyte maturation and stimulates the process of immunoglobulin (Ig) production by those cells [6]. It was also noted that BAFF is responsible for the change in the profile of the secreted immunoglobulins by $B$ cells with IgM and
IgD into IgG, which prolongs the duration of immune response. Therefore, it helps convert an acute inflammation into a chronic process [6]. The role of VEGF is also very well elucidated in the course of rheumatic diseases. The development of ECs and hyperemia of specific organs is an essential element of an ongoing inflammatory process. VEGF assists in spreading this process into other healthy tissues [7]. The VEGF production is possible thanks to keratinocytes, ECs, macrophages, and others [8].

The aim of the study involves the assessment and comparative analysis of the concentrations of $\mathrm{IL}-35$, TNF- $\alpha$, BAFF, and VEGF in peripheral blood serum in patients with rheumatoid arthritis (RA), systemic lupus erythematosus (SLE), antiphospholipid syndrome (APS), and mixed connective tissue disease (MCTD), compared to healthy individuals. The obtained results may constitute not only complementary information concerning the participation of inflammatory cytokines in the pathophysiology of the most common rheumatic diseases, but they may also serve as a prognostic tool for clinicians at early stages of diagnostic work-up. The obtained data and analysis may also facilitate the development of new targeted immune therapies depending on the diagnosed rheumatic disease. According to the present authors' best knowledge, professional literature available worldwide does not include a similar research paper that presents a comprehensive overview of the participation of those cytokines in various rheumatic diseases.

\section{Material and methods \\ Study population}

The study population included 43 patients with RA, 28 patients with SLE, 24 with APS, and nine with MCTD. The study was approved by the Research Ethics Committee of the National Institute of Geriatrics, Rheumatology and Rehabilitation in Warsaw.

All the subjects included in the study were of European descent. SLE, RA, APS, and MCTD patients were recruited from the Connective Tissue Diseases Department of the National Institute of Geriatrics, Rheumatology and Rehabilitation in Warsaw, Poland. All patients signed an informed consent form, and clinical data were collected from patient files and questionnaires. The clinical characteristics of all patients are presented in Table I.

All RA patients fulfilled the 1987 American College of Rheumatology (ACR) or the 2010 EULAR/ACR classification criteria for RA [9]. Most of the RA patients were classified as a group with high disease activity with an average DAS-28 of 5.29 (2.21-7.7). SLE patients fulfilled the 1982 revised American College of Rheumatology classification criteria for SLE [10]. All APS patients fulfilled the preliminary criteria for the classification of 
Table I. Characteristics of the study groups

\begin{tabular}{|c|c|c|c|c|}
\hline Characteristic & $\begin{array}{c}\text { APS } \\
(n=24)\end{array}$ & $\begin{array}{c}\text { SLE } \\
(n=28)\end{array}$ & $\begin{array}{l}\text { MCTD } \\
(n=9)\end{array}$ & $\begin{array}{c}\text { RA } \\
(n=43)\end{array}$ \\
\hline Age & $42(19-68)$ & 34 (19-74) & $35(19-60)$ & $60(32-75)$ \\
\hline Female $(n[\%])$ & $14(58)$ & $22(78)$ & $7(78)$ & $38(88)$ \\
\hline Disease duration (years) & $1(0-16)$ & $2(0-29)$ & $7(1-34)$ & $8(0-34)$ \\
\hline CRP (mg/l) & $10(1-82)$ & $4(1-84)$ & $5(1-8)$ & $13(3-11)$ \\
\hline ESR $(\mathrm{mm} / \mathrm{h})$ & $20(2-63)$ & $22(2-79)$ & $11(2-105)$ & $36(6-160)$ \\
\hline Thrombocytopenia (\%) & 17 & 14 & 33 & 5 \\
\hline Leukocytopenia (\%) & 9 & 40 & 11 & 2 \\
\hline CAD (\%) & 13 & $25(n=24$ total $)$ & 33 & 26 \\
\hline Serology & $\begin{array}{c}\text { Anti-dsDNA (+) 39\% } \\
\text { Anti-histon (+) } 24 \% \\
\text { Anti-Ro (+) 29\% } \\
\text { Anti-La (+) 12\% } \\
\text { Anti-Scl-70 (+) 12\% } \\
\text { Anti-U1-RNP (+) 6\% }\end{array}$ & $\begin{array}{c}\text { Anti-dsDNA (+) 75\% } \\
\text { Anti-histon (+) 29\% } \\
\text { Anti-Ro (+) } 46 \% \\
\text { Anti-La (+) 25\% } \\
\text { Anti-Sm (+) 21\% } \\
\text { Anti-U1-RNP (+) 1\% }\end{array}$ & $\begin{array}{c}\text { Anti-CENP-B (+) 22\% } \\
\text { Anti-U1-RNP (+) 100\% } \\
\text { Anti-U1-70 kD (+) 47\% } \\
\text { A (+) 29\% } \\
\text { C (+) } 18 \%\end{array}$ & $\begin{array}{c}\text { Anti-CCP (+) 79\% } \\
\text { Anti-RF (+) 64\% }\end{array}$ \\
\hline
\end{tabular}

APS - antiphospholipid syndrome, SLE - systemic lupus erythematosus, MCTD - mixed connective tissue disease, RA - rheumatoid arthritis.

catastrophic APS [11]. Mixed connective tissue disease patients fulfilled the preliminary diagnostic criteria for the classification for MCTD [12]. The average score the Systemic Lupus Erythematosus Disease Activity Index (SLEDAI) in patients with SLE and MCTD was 6 and 7, respectively.

Assays for serum levels of IL-35, TNF- $\alpha$, BAFF, and VEGF. Serum samples were separated from peripheral venous blood and collected at $-86^{\circ} \mathrm{C}$ until analysis. Commercially available enzyme-linked immunosorbent assay (ELISA) kits were used for measuring of IL-35, TNF- $\alpha$, BAFF, and VEGF levels in serum of RA, SLE, APS, and MCTD patients. VEGF, TNF- $\alpha$, and BAFF levels were determined using, respectively, a human VEGF ELISA kits, human TNF- $\alpha$ Immunoassay, and Human BAFF/BLyS/ TNFSF13B Quantikine ELISA Kit (R\&D systems, Minneapolis, MN, USA), according to the manufacturer's instructions. The minimum level of detection for VEGF was $9 \mathrm{pg} / \mathrm{ml}$. The minimum level of detection for TNF- $\alpha$ was $5.5 \mathrm{pg} / \mathrm{ml}$, BAFF - $6.44 \mathrm{pg} / \mathrm{ml}$. IL-35 level was determined by Human IL-35 ELISA kit (EH3273, Wuhan Fine Biotech Co. Ltd., East Like High-tech Development District, Wuhan, China). The sensitivity of the test was $<9.375 \mathrm{pg} / \mathrm{ml}$. The plates were read using an LT-4000 MicroPlate Reader (LabTech, USA).

\section{Statistical analysis}

Shapiro-Wilk test was used to check data normality distribution. Kruskal-Wallis test and Dunn's Multiple Comparison test were used to compare differences of serum cytokine levels among the analyzed groups. Spearman's correlation test was used to assess the association between cytokines. A two-tailed $p$-value of less than 0.05 was considered as statistically significant.

\section{Results}

Significant differences in VEGF concentrations occurred between patients with APS and RA, and RA compared with SLE ( $p=0.0005)$ (Fig. 1). Moreover, in RA patients VEGF serum concentrations were the highest (average $330 \mathrm{pg} / \mathrm{ml}$ ) whereas in SLE patients they were almost seven times lower. In patients with RA significant, moderate, positive correlation was noted between VEGF and BAFF concentration $(r=0.5, p=0.02)$.

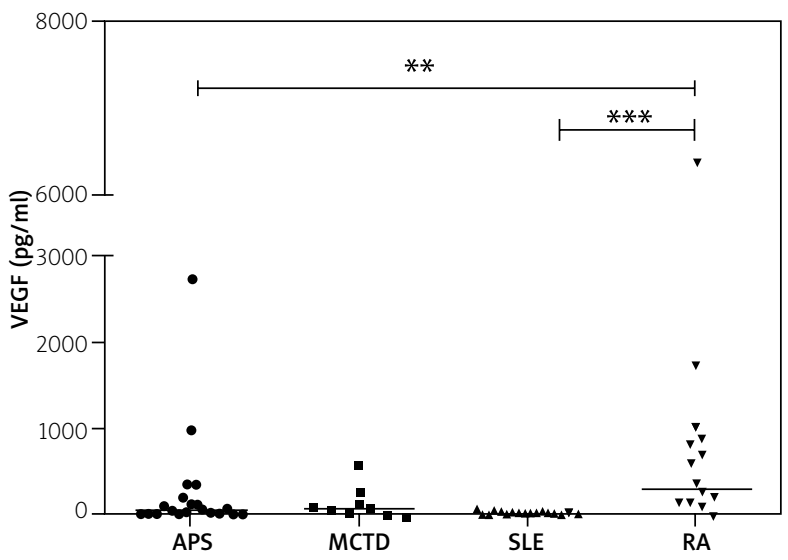

Fig. 1. Serum VEGF $(\mathrm{pg} / \mathrm{ml})$ in APS, MCTD, SLE and RA patients. Kruskal-Wallis and Dunn's Multiple comparison test. 


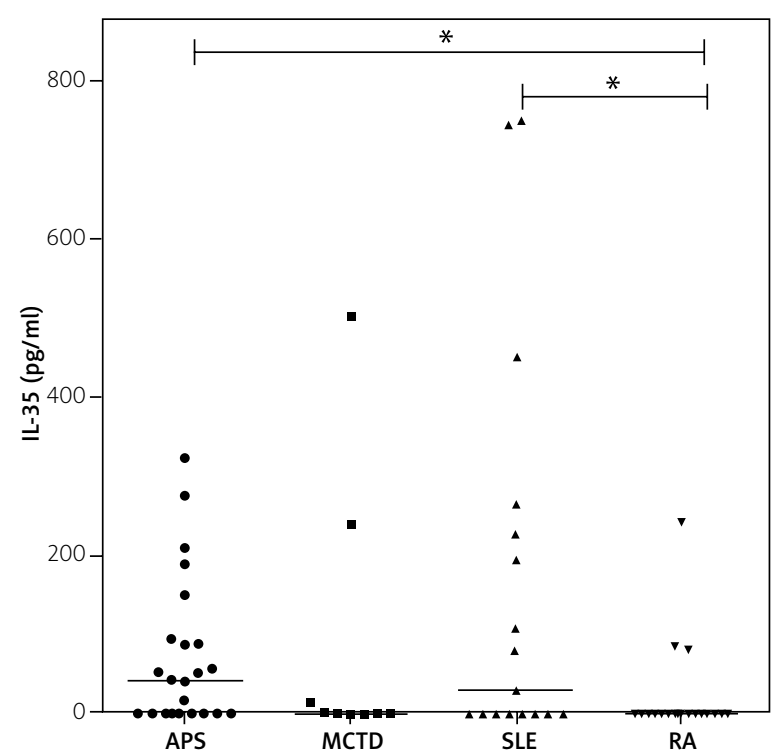

Fig. 2. Serum IL-35 (pg/ml) in APS, MCTD, SLE and RA patients. Kruskal-Wallis and Dunn's multiple comparison test.

Statistically significant differences in IL-35 concentration occurred also between RA patients and patients with APS and SLE (Fig. 2). Nevertheless, the highest average concentration was observed in APS patients. In the patients with SLE a very broad range of concentrations was observed (246 [0-749] pg/ml).

There were no statistically significant differences in BAFF concentrations between the studied groups $(p=0.09)$. The highest concentration was revealed in APS (806 [377-1557] pg/ml) whereas the lowest was in RA (631 [302-3678] pg/ml). However, the maximal level of BAFF was noted in RA patients. Furthermore, in APS significant and strong correlation $(r=0.77, p<0.00)$ between BAFF and TNF- $\alpha$ was noted (Table II), and similar, moderate correlation $(r=0.55, p=0.00)$ was revealed in SLE patients (Table III).

TNF- $\alpha$ was mostly not detected in SLE and MCTD patients. $83 \%$ of RA patients revealed TNF- $\alpha$ below detection level.

\section{Discussion}

The pathophysiology of rheumatic diseases is still not fully understood. It is known that inflammatory cytokines and growth factors (including IL-35, TNF- $\alpha$, BAFF, and VEGF) play key roles in the etiopathogenesis of autoimmune diseases. So far, most of the research has focused on the analysis of the role of individual proinflammatory factors in the development of rheumatic diseases. Therefore, the aim of this study is a comparative analysis and assessment of correlation of the con-

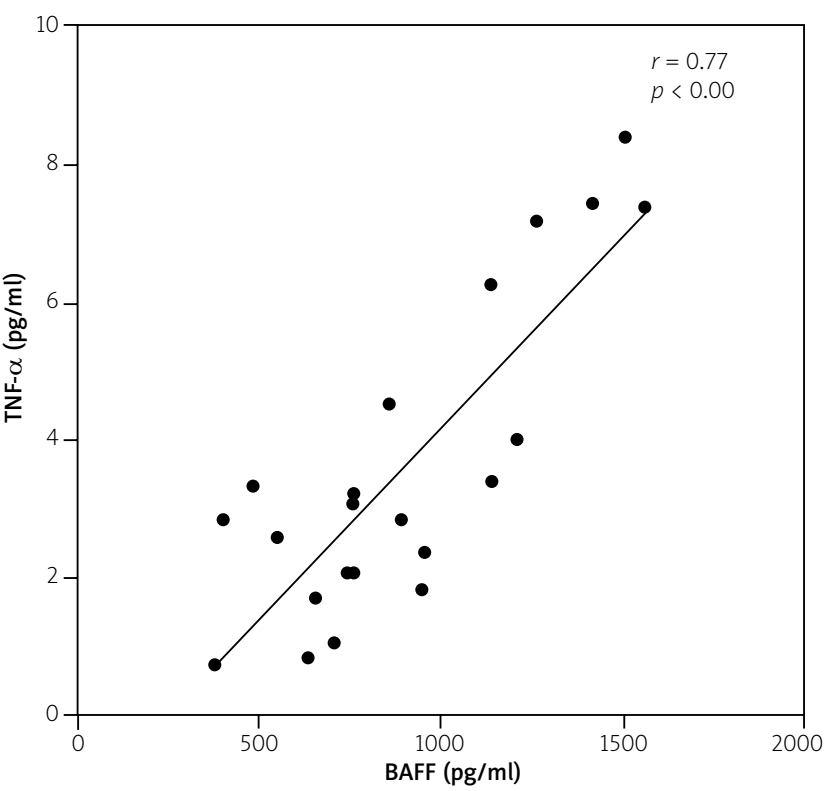

Fig. 3. Serum TNF- $\alpha(\mathrm{pg} / \mathrm{ml})$ correlation with BAFF (pg/ml) in APS patients. Spearman's correlation test.

centrations of several cytokines in the most commonly occurring immune-mediated diseases such as RA, SLE, APS, and MCTD. The intention of the authors is to predict the factors for further laboratory analysis in order to search for biomarkers for specific rheumatic diseases. As a result, it may be also possible to more accurately assess the potential impact of the cytokines and growth factors on the development of this group of diseases.

Nevertheless, the present result must be considered with caution. The first limitation is the small number of patients in the MCTD group. The second one concerns the age of RA patients, which is higher in comparison with the average age in the other analyzed groups. Moreover, the difference in medical treatment should be addressed in future research.

The present study revealed variability of selected cytokine concentrations in patients with different rheumatic diseases. Statistically significant differences were observed in the case of VEGF and IL-35. Additionally, high positive correlation between BAFF and TNF- $\alpha$ in APS patients was observed.

The highest serum concentration of VEGF was noted in RA patients. The present results are in line with many other studies [13-15]. Smets et al. [16] revealed higher VEGF concentration in the serum of elderly patients with rheumatic diseases ( $>60$ years old) such as RA, polymyalgia rheumatica (PMR), giant cell arteritis (GCA), and remitting symmetrical seronegative synovitis with pitting edema (RS3PE), relative to controls. However, the differences in VEGF serum concentration and plasma 
Table II. Correlation of serum cytokines level in APS patients. Significance at $p<0.05$

\begin{tabular}{|c|c|c|c|c|}
\hline Value & BAFF & IL-35 & VEGF & TNF- $\alpha$ \\
\hline \multicolumn{5}{|l|}{ APS $r$} \\
\hline BAFF & & 0.026489 & 0.187863 & 0.7756917 \\
\hline IL-35 & 0.02648894 & & -0.25647 & 0.104937 \\
\hline VEGF & 0.1878629 & -0.25647 & & 0.2923782 \\
\hline TNF- $\alpha$ & 0.7756917 & 0.104937 & 0.292378 & \\
\hline$p$-value & BAFF & IL-35 & VEGF & TNF- $\alpha$ \\
\hline BAFF & & 0.904505 & 0.414798 & $<0.0000$ \\
\hline IL-35 & 0.9045048 & & 0.261758 & 0.6337081 \\
\hline VEGF & 0.4147979 & 0.261758 & & 0.198399 \\
\hline TNF- $\alpha$ & $<0.0000$ & 0.633708 & 0.198399 & \\
\hline
\end{tabular}

APS - antiphospholipid syndrome, BAFF - B-cell-activating factor, IL-35 - interleukin 35, VEGF-vascular endothelial growth factor, TNF- $\alpha$-tumour necrosis factor $\alpha$.

between patients with RS3PE, RA, PMR, and GCA were not significant [16]. Hence, Smets et al. questioned earlier indications that VEGF could be a marker of specific rheumatoid diseases [17], which might be controversial in light of the presented outcomes.

Interestingly, it is still uncertain whether VEGF is only an inflammatory factor or is responsible for the period of tissue regeneration in various rheumatic diseases such as RA. Yoo et al. [18] indicated that VEGF stimulates secretion of inflammatory cytokines in rheumatic diseases. On the other hand, Roy et al. [19] drew attention to the potential participation of VEGF in promoting anabolic and anti-inflammatory phenomena.

In the present study, IL-35 was determined at the lowest level in RA patients, which is in agreement with other research [20]. It is connected to recent studies confirming the role of IL-35 in inhibiting processes associated with VEGF/Ang2/Tie2 pathway [21]. This observation correlates with the results of our study, in which patients with RA are characterized by the highest concentration of VEGF and the lowest of IL-35. Blocking IL-35 activity presented in RA stimulates neovascularization and, potentially, inflammatory processes during the acute phase of the disease. Subsequent results of our study indicate that potentially protective cytokines, such as IL-35, have the highest concentrations in APS, followed by SLE patients. Recently, Álvarez-Rodríguez et al. [22] also found that the level of IL-35 in serum in APS is significantly higher in comparison to that seen in healthy controls. Moreover, in the abovementioned study, in the case of patients with SLE, a lower serum level trend was revealed, and the IL-35 concentration was comparable with healthy controls [22].

The concentrations of BAFF did not differ significantly in the studied groups; therefore, it seems that its value as a biomarker for specific rheumatic diseases is rather
Table III. Positive of serum cytokines level in SLE patients. Significance at $p<0.05$

\begin{tabular}{lcccc}
\hline Value & BAFF & IL-35 & VEGF & TNF- $\alpha$ \\
\hline SLE $r$ & & & & \\
\hline BAFF & & -0.15 & 0.01 & 0.55 \\
\hline IL-35 & -0.15 & & -0.02 & -0.29 \\
\hline VEGF & 0.01 & -0.02 & & 0.04 \\
\hline TNF- $\alpha$ & 0.55 & -0.29 & 0.04 & \\
\hline$p$-value & & & & \\
\hline BAFF & & 0.62 & 0.95 & 0.00 \\
\hline IL-35 & 0.62 & & 0.91 & 0.10 \\
\hline VEGF & 0.95 & 0.91 & & 0.71 \\
\hline TNF- $\alpha$ & 0.00 & 0.10 & 0.71 & \\
\hline SLE - system & & &
\end{tabular}

SLE - systemic lupus erythematosus, BAFF-B-cell-activating factor, IL-35-interleukin 35, VEGF-vascular endothelial growth factor, TNF- $\alpha$ - tumour necrosis factor $\alpha$.

questionable. However, an interesting result is the observation of a positive correlation between the concentration of BAFF and TNF- $\alpha$ in APS. In the case of SLE the same trend was observed (moderate correlation). This result may indicate the existence of a very similar etiopathogenesis of these diseases at the cellular level. The relationship between TNF- $\alpha$ and activation of immune cells, including B cells, is quite well understood [23]. It is interesting that a similar correlation is not noticeable in the other groups we studied. Certainly, this requires further research, especially on numerous groups of patients and clinically homogeneous populations. Similarly, patients with MCTD require more extensive research.

In the present study, some statistical convergence was noticed in this group of patients, especially in relation to VEGF concentrations in APS. Probably, due to 
the small number of patients in the MCTD group $(n=9)$, statistical significance was not reached.

\section{Conclusions}

Inflammatory cytokines and growth factors play a key role in the etiopathogenesis of rheumatic diseases. Promising biomarkers in patients with APS are BAFF and TNF- $\alpha$. Further study of the significance of VEGF in RA pathogenesis is still required. IL-35 may be a useful marker for the diagnosis of APS. At the same time, IL-35 appears to be the least specific for RA patients. Some similar statistical trends were observed in MCTD and APS patients, especially in relation to VEGF concentrations. To determine if there is some statistical significance between these two study groups, further study of inflammatory cytokines and growth factor profiles on a larger MCTD group is necessary. Positive correlation of BAFF and TNF- $\alpha$ concentrations in APS and SLE potentially indicates much more similar etiopathogenesis of these diseases than could previously be predicted.

Considering the complexity of the pathophysiology of rheumatic diseases, further research is still needed, especially on larger, clinically unified populations.

The authors declare no conflict of interest.

\section{References}

1. Aringer M, Schulze-Koops H. Immunology of systemic inflammatory diseases. Orthopade 2018; 47: 891-897.

2. Raciborski F, Kłak A, Kwiatkowska B, et al. Diagnostic delays in rheumatic diseases with associated arthritis. Reumatologia 2017; 55: 169-176.

3. Anaya JM, Shoenfeld Y, Buttgereit F, Gonzalez-Gay MA. Autoimmune rheumatic diseases. Biomed Res Int 2014; 2014: 952159.

4. Choi J, Leung PS, Bowlus C, Gershwin ME. IL-35 and Autoimmunity: a Comprehensive Perspective. Clin Rev Allergy Immunol 2015; 49: 327-332.

5. Criscione LG, St Clair EW. Tumor necrosis factor-alpha antagonists for the treatment of rheumatic diseases. Curr Opin Rheumatol 2002; 14: 204-211.

6. Lied GA, Berstad A. Functional and clinical aspects of the B-cell-activating factor (BAFF): a narrative review. Scand J Immunol 2011; 73: 1-7.

7. Holmes DI, Zachary I. The vascular endothelial growth factor (VEGF) family: angiogenic factors in health and disease. Genome Biol 2005; 6: 209.

8. Claesson-Welsh L. VEGF receptor signal transduction - A brief update. Vascul Pharmacol 2016; 86: 14-17.

9. Aletaha D, Neogi T, Silman AJ, et al. 2010 rheumatoid arthritis classification criteria: an American College of Rheumatology/ European League Against Rheumatism collaborative initiative. Ann Rheum Dis 2010; 69: 1580-1588.
10. Tiao J, Feng R, Carr K, et al. Using the American College of Rheumatology (ACR) and Systemic Lupus International Collaborating Clinics (SLICC) criteria to determine the diagnosis of systemic lupus erythematosus (SLE) in patients with subacute cutaneous lupus erythematosus (SCLE). I Am Acad Dermatol 2016; 74: 862-869.

11. Asherson RA, Cervera R, de Groot PG, et al. Catastrophic antiphospholipid syndrome: International consensus statement on classification criteria and treatment guidelines. Lupus 2003; 12: 530-534.

12. Kasukawa R, Too T, Miyawaki S, et al. Preliminary diagnostic criteria for classification of mixed connective tissue disease. In: Mixed connective tissue diseases and anti-nuclear antibodies, Kasukawa R, Sharp GC (eds.). Elsevier, Amsterdam 1987: $41 \mathrm{e} 7$

13. Harada M, Mitsuyama K, Yoshida $\mathrm{H}$, et al. Vascular Endothelial Growth Factor in Patients with Rheumatoid Arthritis. Scand J Rheumatol 1998; 27: 377-380.

14. Ballara S, Taylor PC, Reusch P, et al. Raised serum vascular endothelial growth factor levels are associated with destructive change in inflammatory arthritis. Arthritis Rheum 2001; 44: 2055-2064.

15. Lee SS, Joo YS, Kim WU, et al. Vascular endothelial growth factor levels in the serum and synovial fluid of patients with rheumatoid arthritis. Clin Exp Rheumatol 2001; 19: 321-324.

16. Smets P, Devauchelle-Pensec V, Rouzaire PO, et al. Vascular endothelial growth factor levels and rheumatic diseases of the elderly. Arthritis Res Ther 2016; 18: 283.

17. Arima K, Origuchi T, Tamai M, et al. RS3PE syndrome presenting as vascular endothelial growth factor associated disorder. Ann Rheum Dis 2005; 64: 1653-1655.

18. Yoo SA, Kwok SK, Kim WU. Proinflammatory role of vascular endothelial growth factor in the pathogenesis of rheumatoid arthritis: prospects for therapeutic intervention. Mediators Inflamm 2008; 2008: 129873

19. Roy H, Bhardwaj S, Ylä-Herttuala S. Biology of vascular endothelial growth factors. FEBS Lett 2006; 580: 2879-2887.

20. Ning X, Jian Z, Wang W. Low Serum Levels of Interleukin 35 in Patients with Rheumatoid Arthritis. Tohoku J Exp Med 2015; 237: 77-82.

21.Jiang S, Li Y, Lin T, et al. IL-35 Inhibits Angiogenesis through VEGF/Ang2/Tie2 Pathway in Rheumatoid Arthritis. Cell Physiol Biochem 2016; 40: 1105-1116.

22. Álvarez-Rodríguez L, Martínez-Taboada V, Calvo-Alén J, et al. Altered Th17/Treg Ratio in Peripheral Blood of Systemic Lupus Erythematosus but Not Primary Antiphospholipid Syndrome. Front Immunol 2019; 10: 391.

23. Sang M, Li J, Wei Z, et al. Molecular structure, expression, and bioactivity of B-cell-activating factor of the TNF family (BAFF) and its receptor BAFF-R in cats (Felis catus). Mol Immunol 2019; 112: 59-71. 